\title{
Supporting Information: Dynamic color generation with electrically tunable thin film optical coatings
}

Kandammathe Valiyaveedu Sreekanth, a, b Rohit Medwal, c Yogesh Kumar Srivastava a, b,

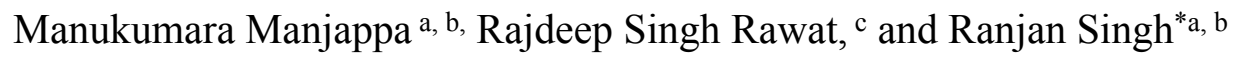

a. Division of Physics and Applied Physics, School of Physical and Mathematical Sciences, Nanyang Technological University, 21 Nanyang Link, Singapore-637371

b. Centre for Disruptive Photonic Technologies, The Photonic Institute, Nanyang Technological University, 50 Nanyang Avenue, Singapore-639798

c. Natural Sciences and Science Education, National Institute of Education, Nanyang Technological University, 1 Nanyang Walk, Singapore 637616

*Correspondence author E-mail: ranjans@,ntu.edu.sg 


\section{Methods}

Sample fabrication. Thin film optical coatings were fabricated on cleaned Si and quartz substrates. $\mathrm{Ge}_{2} \mathrm{Sb}_{2} \mathrm{Te}_{5}, \mathrm{Sb}_{2} \mathrm{~S}_{3}$, and $\mathrm{Sb}_{2} \mathrm{Te}_{3}$ thin films were deposited using RF magnetron sputtering (Oerlikon Leybold vacuum). The deposition was carried out at room temperature under high purity argon $(99.999 \%)$ at a deposition rate of $0.2 \mathrm{~A} / \mathrm{s}$. Ag and Ge thin films were deposited by thermal evaporation (Oerlikon Leybold vacuum) of $\mathrm{Ag}$ and Ge pellets at deposition rates of $0.2 \mathrm{~A} / \mathrm{s}$, and a common base pressure of $<5 \times 10-6$ mbar.

Microheater fabrication and Temperature calibration: The metallic microheater was fabricated by UV illumination-based photolithography. Thin film of tungsten (W) with a thickness of $200 \mathrm{~nm}$ was thermally deposited over the entire area and followed by a lift-off process to leave behind the $\mathrm{W}$ only in the patterned area. Thereafter, the microheater was subjected to the temperature calibration using Keithley 2450. A thermocouple was mounted at the center of the microheater, and two probes were used to apply the DC current to the heating element. The temperature was recorded as a function of the applied current.

Ellipsometry characterizations. Variable-angle high-resolution spectroscopic ellipsometry (J.A. Woollam Co., Inc., V-VASE) was used to determine the thickness and the optical constants of $\mathrm{Ge}_{2} \mathrm{Sb}_{2} \mathrm{Te}_{5}, \mathrm{Sb}_{2} \mathrm{~S}_{3}, \mathrm{Sb}_{2} \mathrm{Te}_{3}$, Ge, and Ag thin films.

Reflectance and transmission measurements. The normal incidence reflection and transmission measurements were performed using a microspectrophotometer (Jasco, MSV-5200) with a sampling domain size varying from $100 \mu \mathrm{m} \times 100 \mu \mathrm{m}$ to $25 \mu \mathrm{m} \times 25 \mu \mathrm{m}$.

Numerical simulations. Reflection, transmission, and absorption spectra were simulated using transfer matrix method code written in MATLAB. Finite difference time domain (FDTD) method has been used to simulate the intensity field distribution. The commercially available Lumerical 
FDTD software (lumerical.com) was used for this purpose. In the 3D simulation, periodic boundary condition was used along $\mathrm{x}$ and $\mathrm{y}$ directions, and perfectly matched layer (PML) boundary condition was used along $\mathrm{z}$ direction. The plane wave source was used as the incident beam. Experimentally determined complex permittivities of thin films were used in the simulations. 


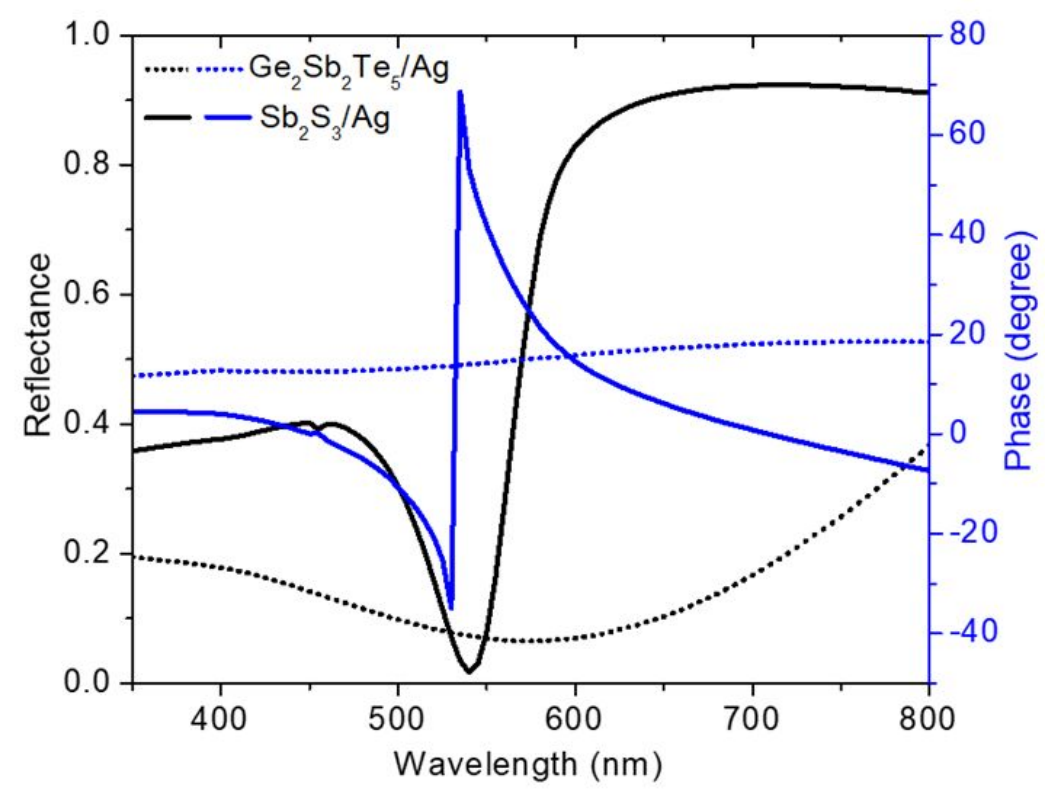

Figure S1 Calculated reflectance and phase spectra of a broadband (GST-Ag) and a narrowband $\left(\mathrm{Sb}_{2} \mathrm{~S}_{3}-\mathrm{Ag}\right)$ absorber. TMM was used to calculate both spectra.
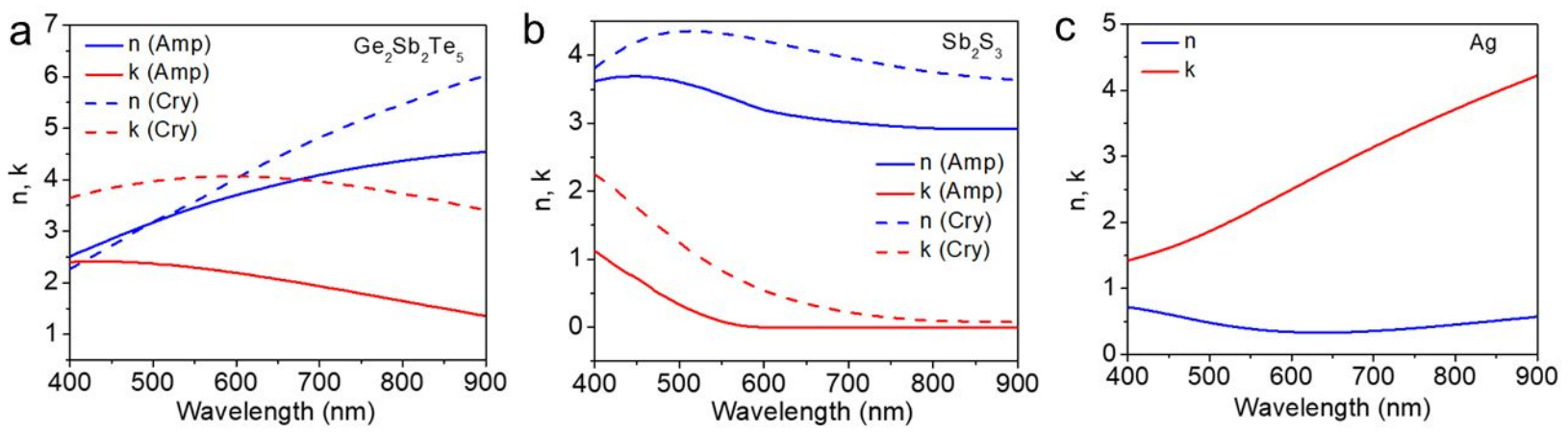

Figure S2 Ellipsometrically determined optical constants of the thin films of (a) GST, (b) $\mathrm{Sb}_{2} \mathrm{~S}_{3}$ and (c) Ag. 


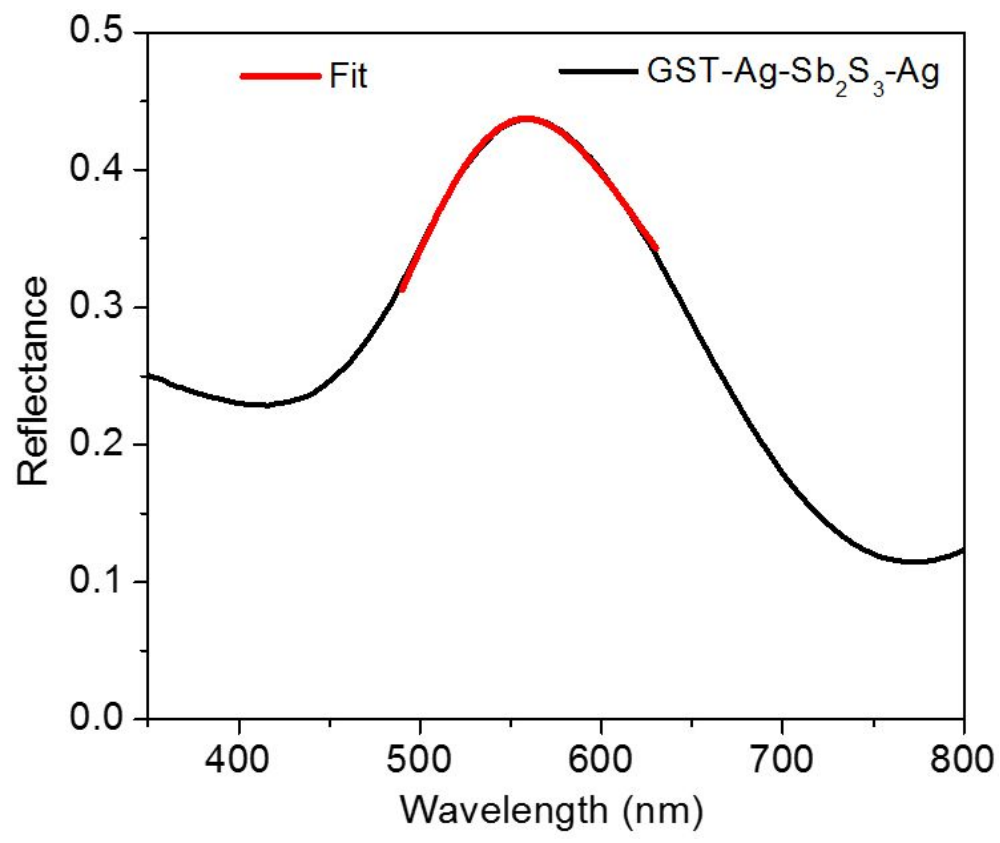

Figure S3 Fano fitting on the measured reflection spectrum of a GST (20 nm)-Ag (20 nm)-Sb $\mathrm{S}_{3}$ $(30 \mathrm{~nm})-\mathrm{Ag}(100 \mathrm{~nm})$ stack. Fano fitting formula is $\sigma(E)=A+D^{2} \frac{(q+\Omega(E))^{2}}{1+\Omega^{2}(E)}$ with $E$ is the energy, $q$ is the Fano parameter, and $\Omega(E)=2\left(E-E_{0}\right) / \Gamma$, where $E_{0}$ and $\Gamma$ are the resonance energy and width. We used the following fitting parameter: $E_{0}=2.3 \mathrm{eV}, \Gamma=5.3 \mathrm{eV}, q=5, A=0$, and $D=0.13$
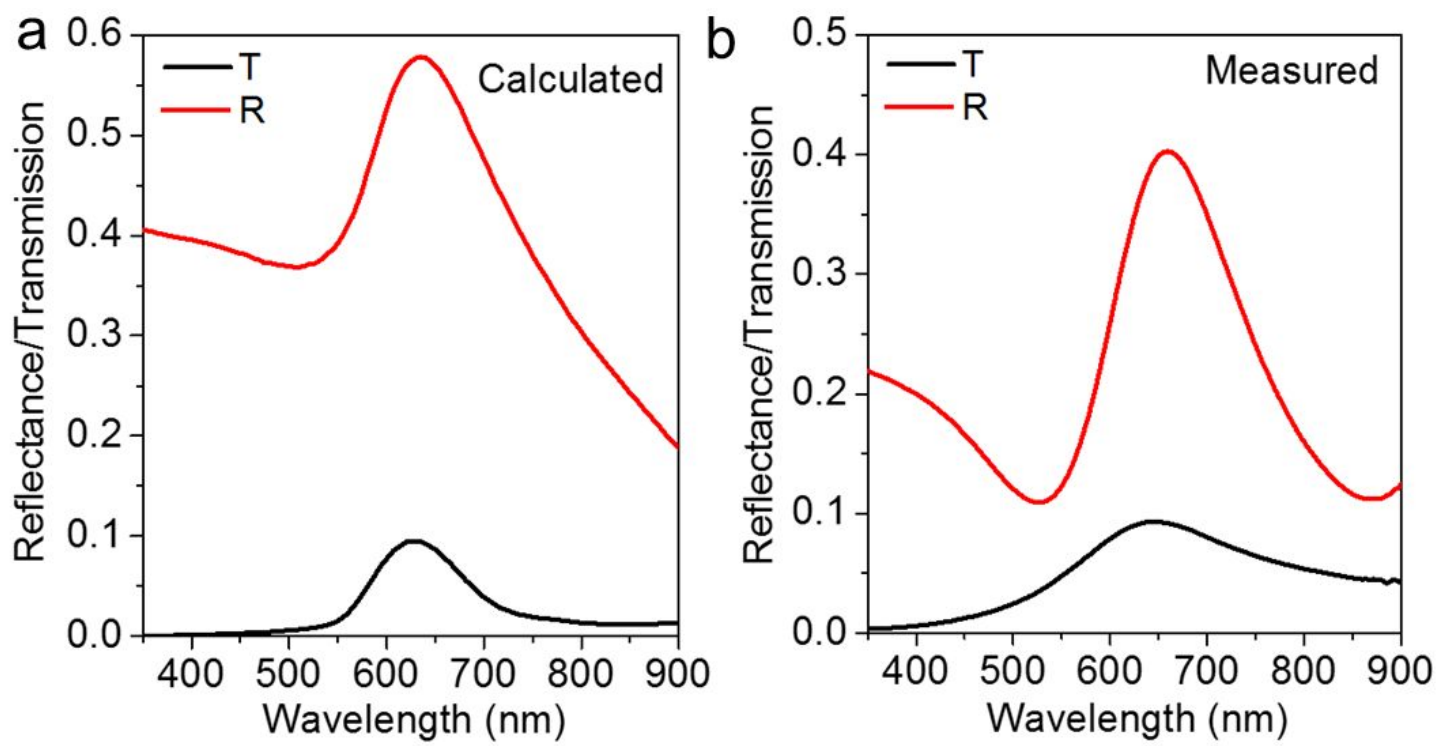

Figure S4 Reflection and transmission spectra of a semitransparent Fano resonant optical coating (GST (20 nm)-Ag (15 nm)-Sb $\left.{ }_{2} \mathrm{~S}_{3}(50 \mathrm{~nm})-\mathrm{Ag}(30 \mathrm{~nm})\right)$ (a) calculated and (b) measured. 

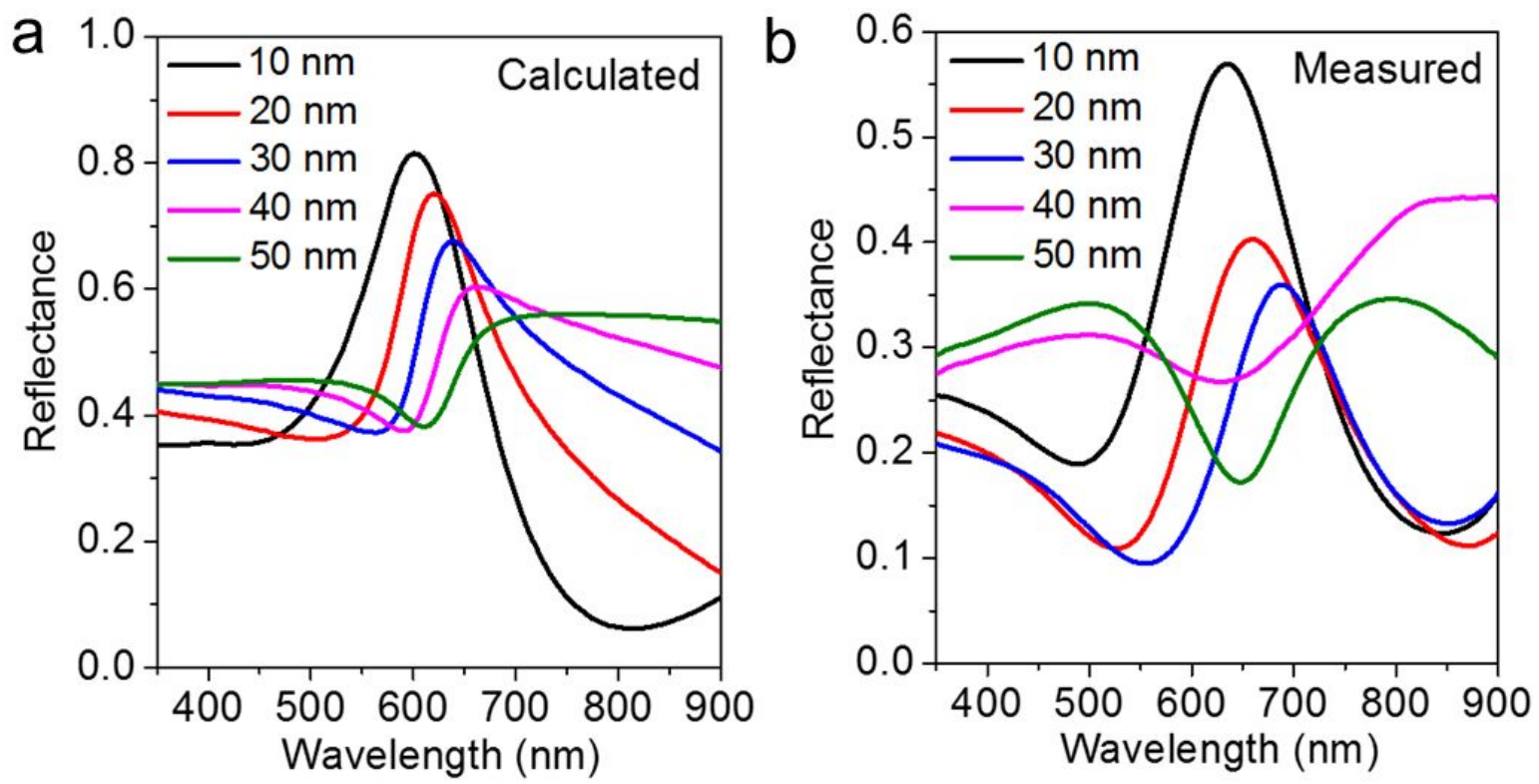

Figure S5 Switching of Fano resonance to conventional cavity resonance by increasing the thickness of GST layer (10 nm to $50 \mathrm{~nm}$ ) (a) calculated and (b) measured.
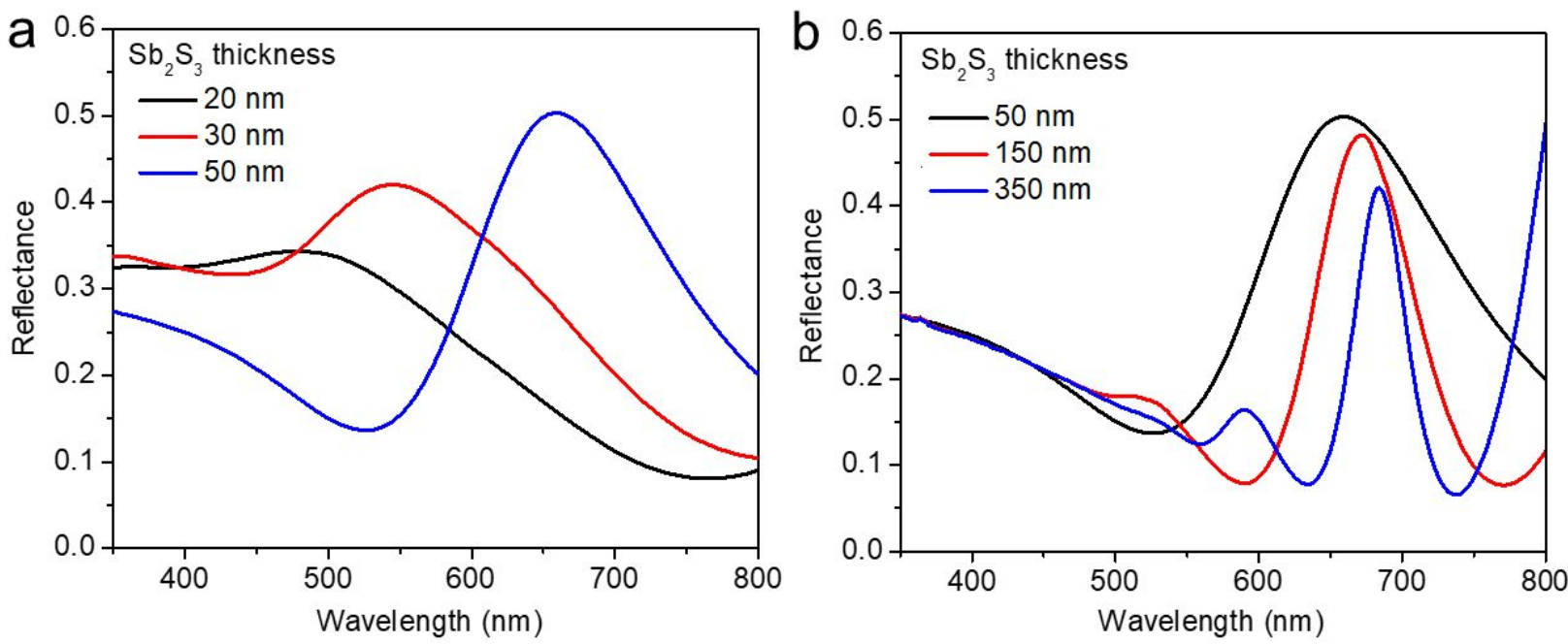

Figure S6 Experimental demonstration of Fano resonance (a) spectral tunability and (b) linewidth narrowing by varying the thickness of $\mathrm{Sb}_{2} \mathrm{~S}_{3}$ cavity layer. 

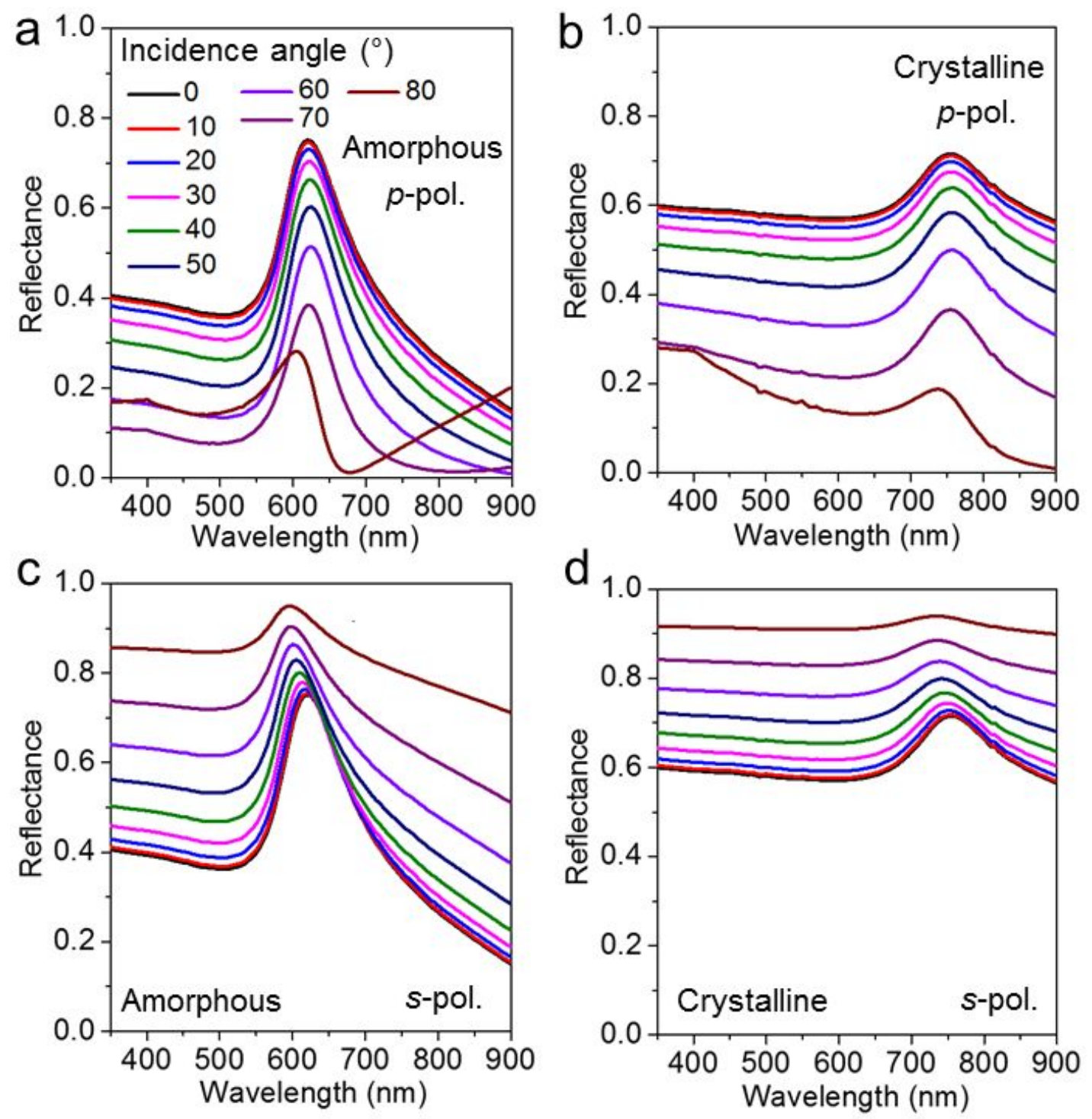

Figure S7 Iridescence-free feature of active Fano resonant optical coatings. Calculated angular reflection spectra of GST $(20 \mathrm{~nm})-\mathrm{Ag}(20 \mathrm{~nm})-\mathrm{Sb}_{2} \mathrm{~S}_{3}(50 \mathrm{~nm})-\mathrm{Ag}(100 \mathrm{~nm})$ stack for (a) $p$ polarization and amorphous phase of PCMs, (b) $p$-polarization and crystallization phase of PCMs, (c) s-polarization and amorphous phase of PCMs, and (d) s-polarization and crystallization phase of PCMs. Fano resonance is polarization and incidence angle ( 0 to 80 degrees) independent for both phases of PCMs in the thin film coating. 

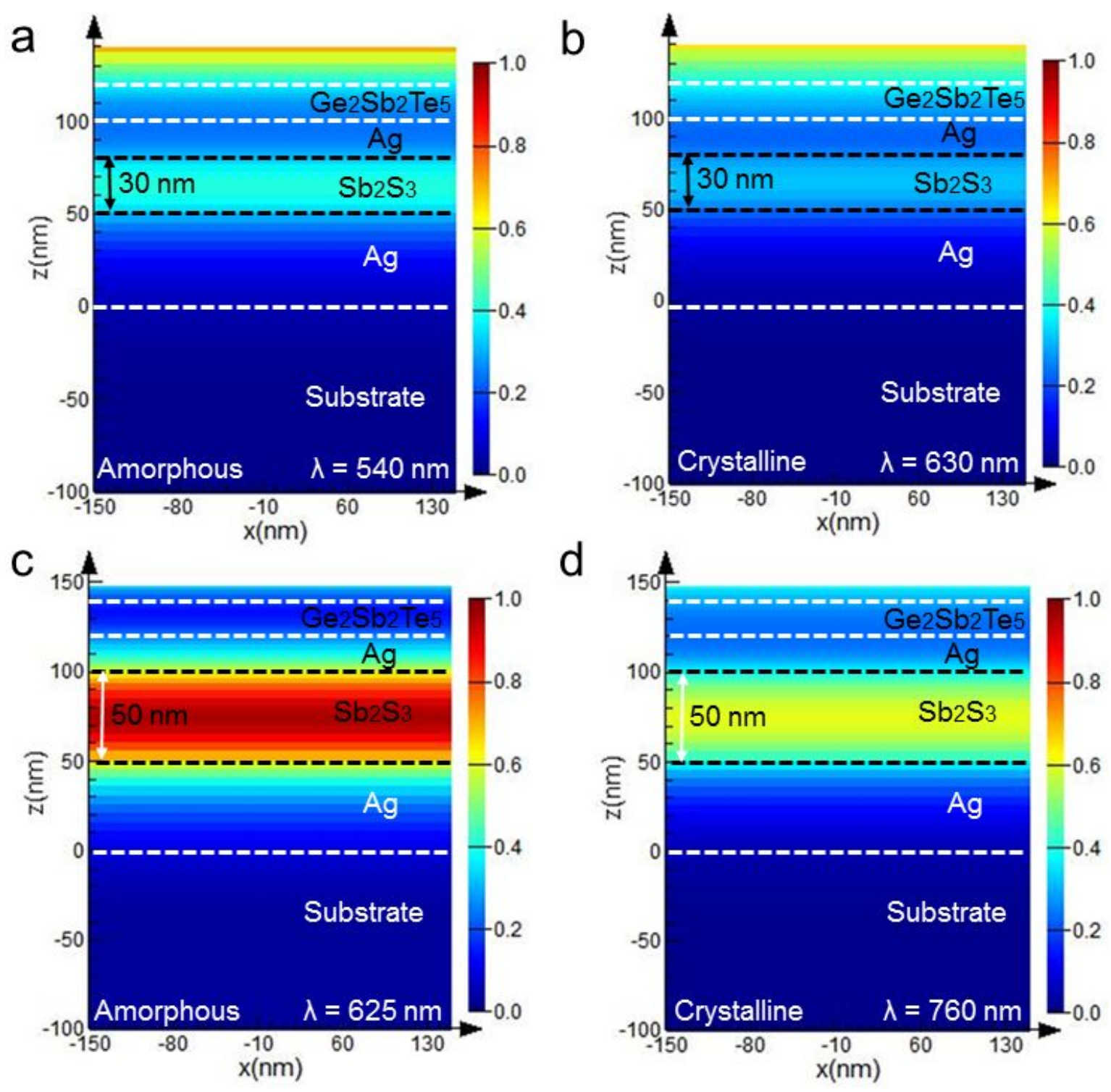

Figure S8 Simulated electric intensity filed distribution at the Fano resonant wavelength for both phases of PCM layers and $\mathrm{Sb}_{2} \mathrm{~S}_{3}$ layer thickness of (a) $30 \mathrm{~nm}$ with amorphous phase at $540 \mathrm{~nm}$, (b) $30 \mathrm{~nm}$ with crystalline phase at $630 \mathrm{~nm}$, (c) $50 \mathrm{~nm}$ with amorphous phase at $625 \mathrm{~nm}$ and (d) 50 $\mathrm{nm}$ with crystalline phase at $760 \mathrm{~nm}$. The intensity is tightly confined in the cavity layer $\left(\mathrm{Sb}_{2} \mathrm{~S}_{3}\right)$ for both phases of PCMs. 

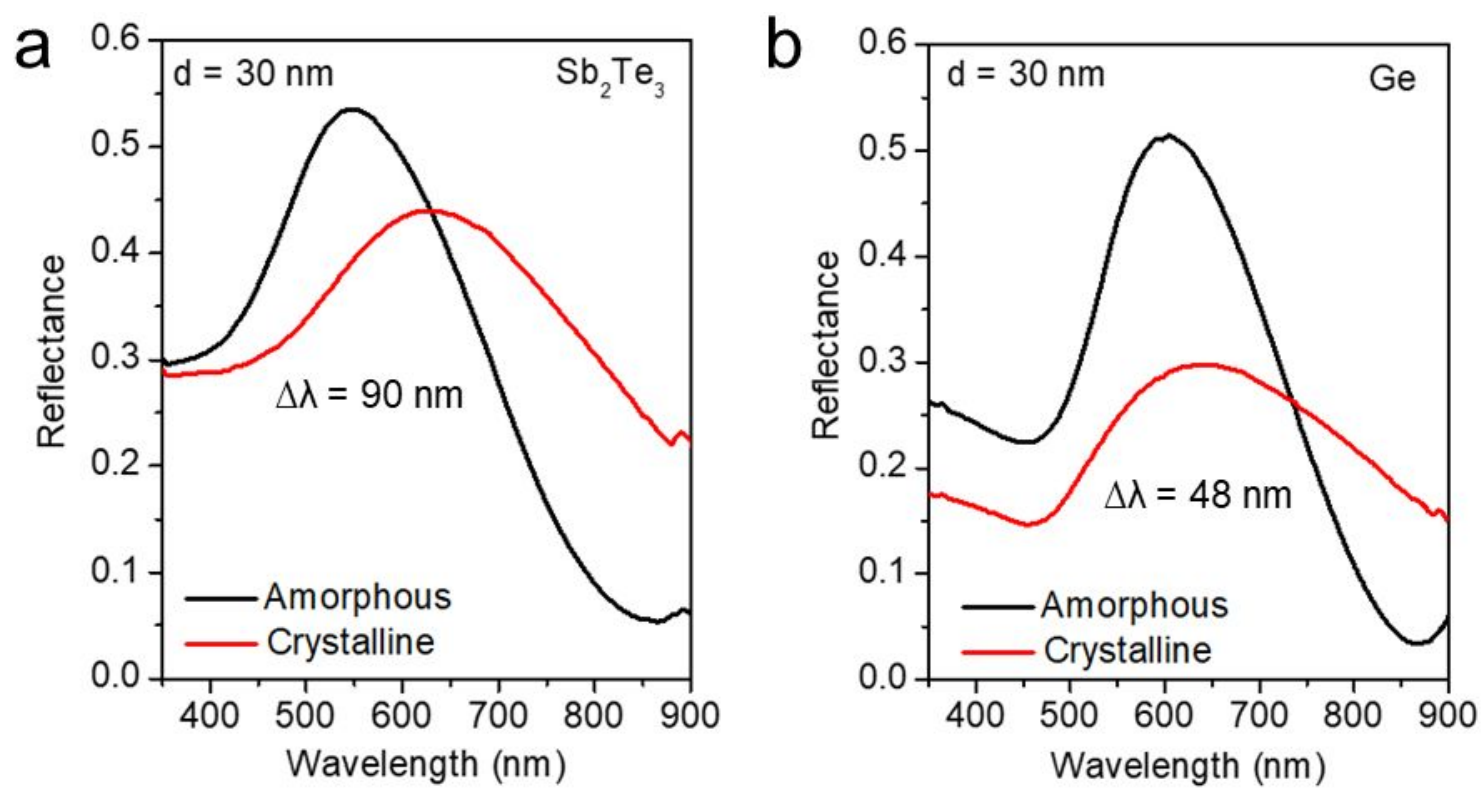

Figure S9 Measured reflection spectra for a Fano resonant optical coating with $30 \mathrm{~nm}$ think $\mathrm{Sb}_{2} \mathrm{~S}_{3}$ layer. Tuning the Fano resonance of GST (20 nm)-Ag (20 nm)-Sb $\mathrm{S}_{3}(30 \mathrm{~nm})-\mathrm{Ag}(100 \mathrm{~nm})$ stack by replacing the top GST layer with a $20 \mathrm{~nm}$ thick layer of (a) $\mathrm{Sb}_{2} \mathrm{Te}_{3}$, and (b) Ge. Almost same spectral shift $(90 \mathrm{~nm})$ and smaller spectral shift $(48 \mathrm{~nm})$ is obtained by replacing another PCM such as $\mathrm{Sb}_{2} \mathrm{Te}_{3}$ and $\mathrm{Ge}$, respectively.
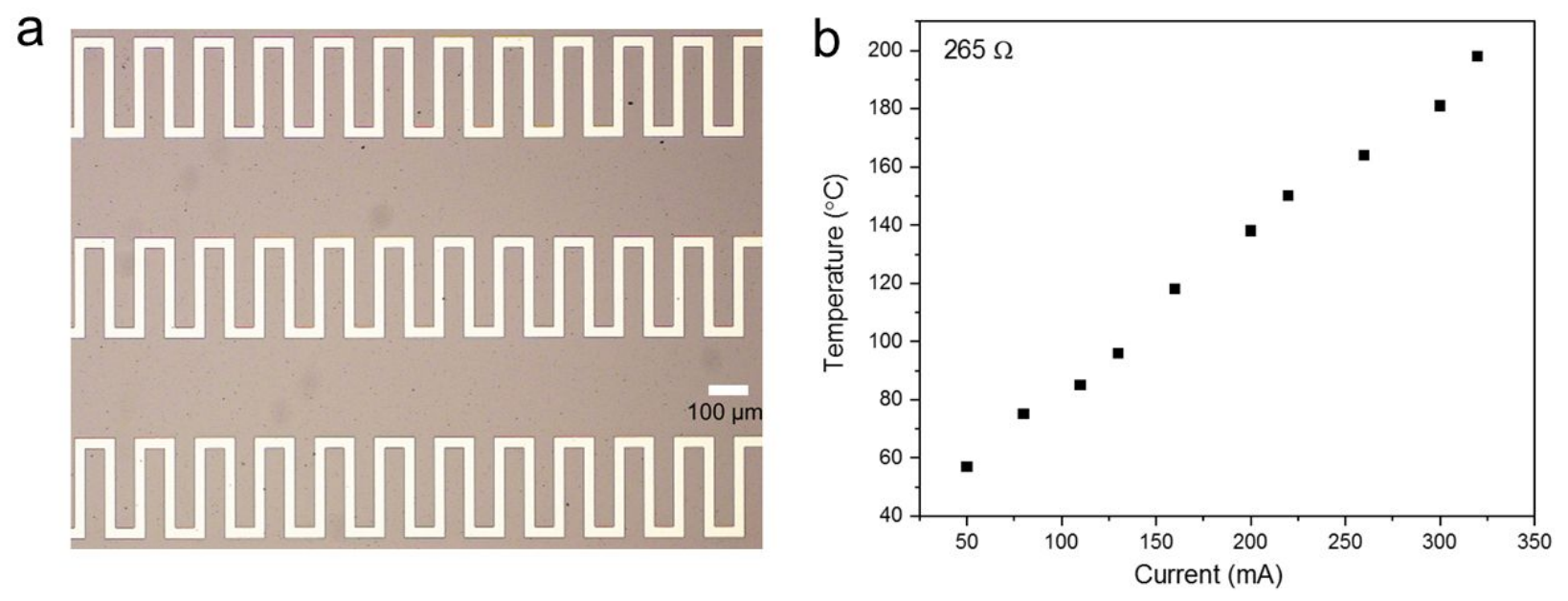

Figure S10 (a) Optical microscopy image of the fabricated microheater/Si device and (b) temperature calibration of the microheater with applied DC current. The measured heater resistance at room temperature is $265 \Omega$. 

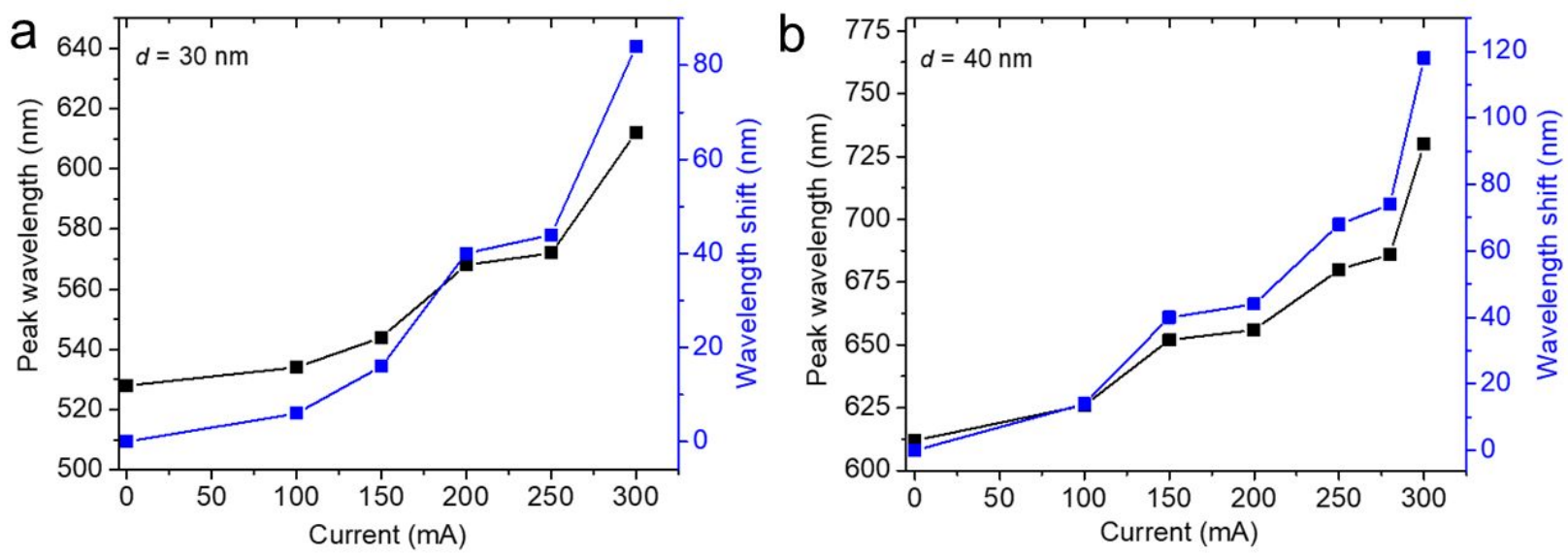

Figure S11 Increasing peak resonance wavelength with increasing current and measured wavelength shift for each current with respect to $0 \mathrm{~mA}$ for different thickness of $\mathrm{Sb}_{2} \mathrm{~S}_{3}$ (a) $d=30$ $\mathrm{nm}$ and (b) $40 \mathrm{~nm}$.
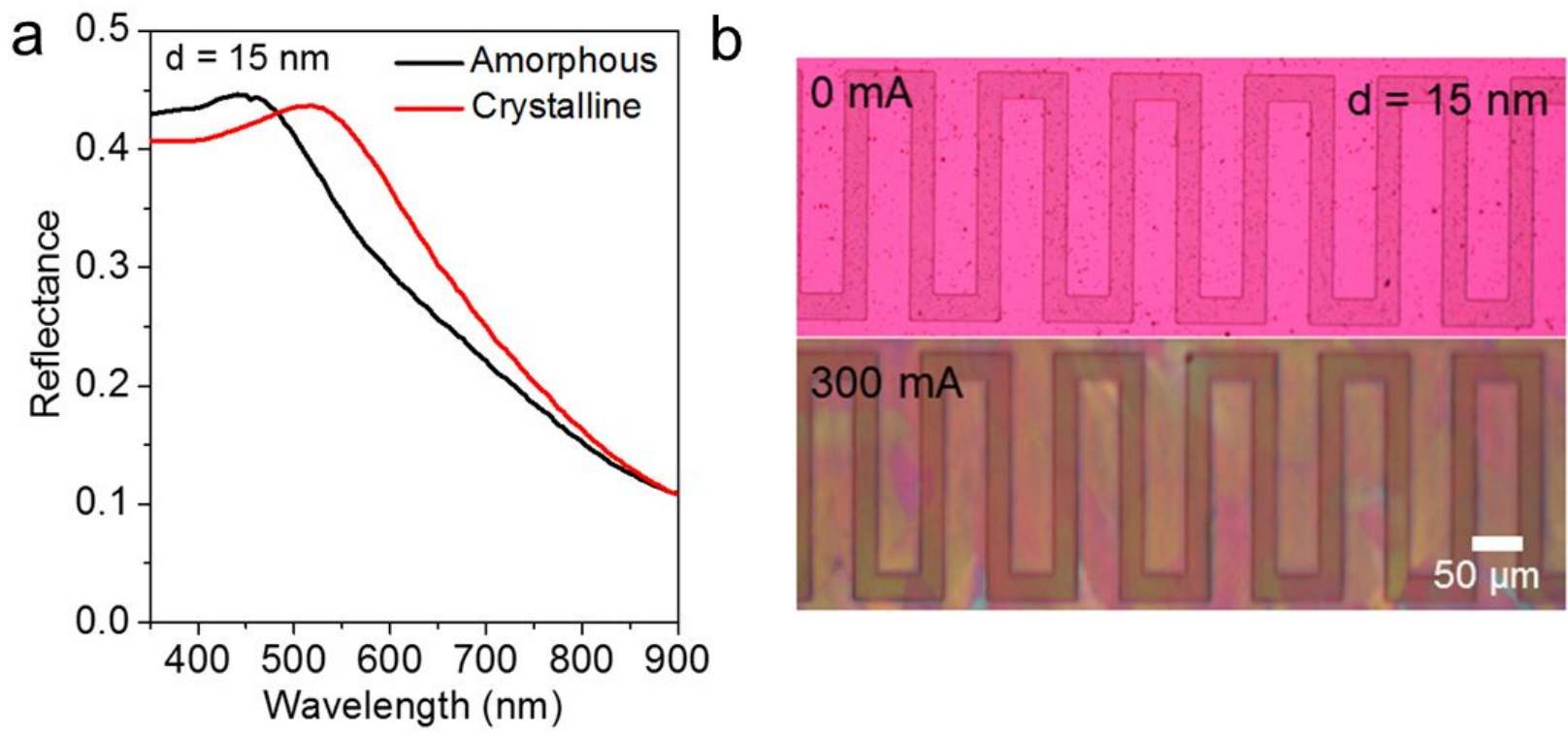

Figure S12 (a) Reflection spectrum of a GST (20 nm)-Ag (20 nm)-Sb $\mathrm{S}_{3}(15 \mathrm{~nm})-\mathrm{Ag}(100 \mathrm{~nm})$ stack in both phases of PCM layers. (b) Optical microscopic image of color change (pink to greenish yellow) observed on Fano resonant optical coating (15 nm thick $\left.\mathrm{Sb}_{2} \mathrm{~S}_{3}\right)$ with applied current of 0 and $300 \mathrm{~mA}$. Here, whole parts of the sample represent Fano resonant coating. 

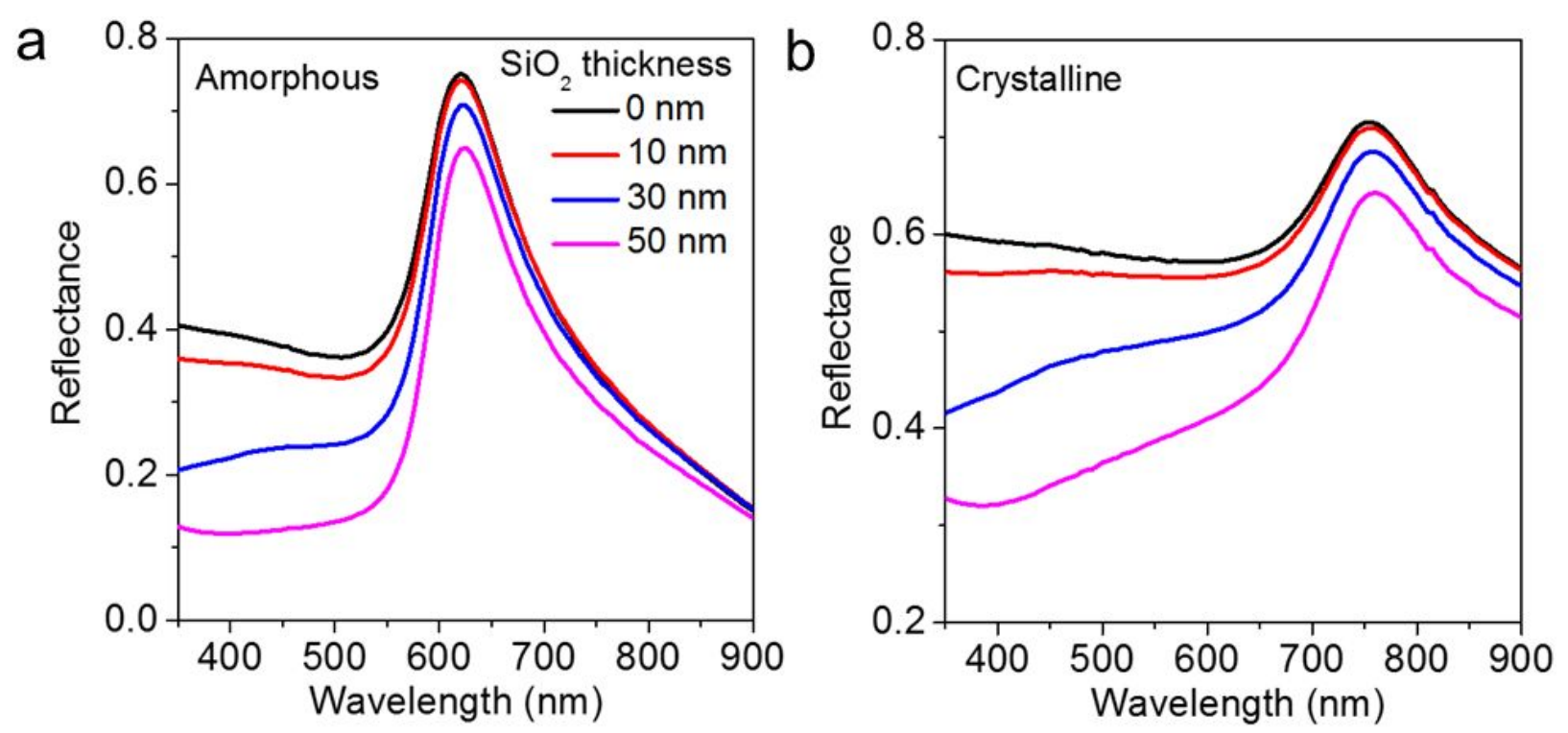

Figure S13 Oxidation-resistance feature of the Fano resonant optical coatings. Reflection spectra of a GST $(20 \mathrm{~nm})-\mathrm{Ag}(20 \mathrm{~nm})-\mathrm{Sb}_{2} \mathrm{~S}_{3}(50 \mathrm{~nm})-\mathrm{Ag}(100 \mathrm{~nm})$ stack with varying thickness of a capping dielectric layer $\left(\mathrm{SiO}_{2}\right)$. PCM layers in (a) amorphous phase and (b) crystalline phase. Significant intensity Fano resonance is obtained even after coating a $\mathrm{SiO}_{2}$ thin layer of thickness of $50 \mathrm{~nm}$. Therefore, the oxidation issue can be overcome by using active Fano thin film coatings. 